Acta Mechanica 93, 133-143 (1992)

\title{
Continuum damage mechanics for softening of brittle materials
}

\author{
W. A. M. Brekelmans, P. J. G. Schreurs and J. H. P. de Vree, Eindhoven, The Netherlands
}

(Received May 23, 1991; revised June 13, 1991)

\begin{abstract}
Summary. Continuum damage theory is used to model the failure behaviour of brittle materials. In the constitutive equations a damage parameter is incorporated. A damage criterion is postulated such that large differences between tension and compression strength can be described. A damage growth law is quantified. based on experimental data for concrete. For the elaboration of the mathematical formulation the finite element method is applied. Numerical results obtained for a plane strain example show the merits of the procedure.
\end{abstract}

\section{Introduction}

The behaviour of brittle materials like concrete under mechanical loads is affected by the development, growth and coalescence of microcracks leading to the formation and propagation of macrocracks and eventually to rupture. Classical fracture mechanics is inappropriate to predict crack-initiation while crack-propagation can only be described when the crack-path is known beforehand. Continuum damage mechanics offers principally more extended possibilities for crack analyses.

The continuum damage approach, first elaborated by Kachanov [1] to model fracture in creep, is characterized by the introduction of separate fields of damage variables which are measures for the local deterioration of the material. By the incorporation of the damage variables into the constitutive relationships the influence of defects on the material behaviour is taken into account.

Micromechanical considerations can be used for the identification and quantification of the damage variables [2]. On the other hand a phenomenological procedure, as will be outlined in this paper, can also be successful. The theory is developed mainly analogous to the usual formulation of elasto-plastic behaviour. A damage criterion (comparable to a "plastic flow criterion") is postulated and a kinetic damage evolution law ("hardening/softening law") will be supplied. Numerical values for model parameters are obtained from experimental data in the literature.

For the detailed analysis of non-homogeneous loaded bodies the finite element method is pre-eminently suitable. The global structure of the particular computational implementation is explained. An application, with promising results, shows the merits of the strategy. 


\section{Constitutive modelling}

Material degradation by increasing microstructural deterioration can be considered as irreversible and therefore as history dependent behaviour. To derive the appropriate constitutive equations, the framework of solid thermodynamics for irreversible processes [3], [4] gives qualitative but useful support. To parameterize history dependence so-called internal hidden variables can be introduced [5], [6]. The following theory will be confined to elastic material behaviour influenced by isotropic damage, denoted by $D$. As viscous or plastic strain contributions are left out of consideration, the damage $D$ is the only relevant internal variable.

The damage $D$ is a monotonously increasing scalar quantity, $0 \leqq D \leqq 1$, expressing the level of material degradation [7]-[9]; initially undamaged material is characterized by $D=0$, the complete loss of stiffness and coherence by $D=1$. As an internal variable, $D$ will contribute to the expression for the Helmholtz free energy (here defined per unit volume) $\Psi(\varepsilon, D)$, with $\varepsilon$ the linear strain tensor. The Cauchy stress tensor $\boldsymbol{\sigma}$ can be derived from the relationship

$\sigma=\sigma(\varepsilon, D)=\frac{\partial \Psi}{\partial \varepsilon}$

Following the usual continuum damage mechanics approach with adoption of the principle of effective stress [10], [11], the expression for $\Psi$ reads

$\Psi=(1-D) \Psi_{0}(\boldsymbol{\varepsilon})$

with $\Psi_{0}(\varepsilon)$ the free energy for undamaged material while $(1-D)$ is the reduction factor caused by damage.

For linear elastic behaviour $\Psi_{0}$ can be written as [12]

$\Psi_{0}(\boldsymbol{\varepsilon})=\frac{1}{2} \varepsilon:{ }^{4} \mathbf{H}: \boldsymbol{\varepsilon}$,

${ }^{4} \mathbf{H}=\frac{v E}{(1+v)(1-2 v)}\left(\mathbf{I I}+\frac{1-2 v}{v}{ }^{4} \mathbf{I}\right)$

with Young's modulus $E$ and Poisson's ratio $v ; \mathbf{I}$ and ${ }^{4} \mathbf{I}$ represent the (symmetric) identity tensors of rank two and four, respectively. The product $(1-D) E$ can be considered as the effective elastic modulus while it is assumed that Poisson's ratio is not affected by damage. Alternative relationships for $\Psi(\varepsilon, D)$ have been proposed, e.g. [13], which also adapt Poisson's ratio; no further attention is given to these formulations.

The constitutive description is completed by a damage criterion and a damage evolution equation. It can be proved that $\dot{D} \geqq 0$ is a necessary condition to satisfy the ClausiusDuhem entropy inequality.

The damage criterion defines the strain states with potential increase of damage. The approach is similar to the procedure followed in the theory of elasto-plasticity. A scalar measure of strain, the damage equivalent strain $\varepsilon_{d}=\varepsilon_{d}(\varepsilon) \geqq 0$, is introduced. An appropriate expression for $\varepsilon_{d}$ will be presented in Section 4 . Increase of damage is only possible if actually $\varepsilon_{d}$ increases and equals a threshold value $\varkappa \geqq 0$, the damage remains constant if $\varepsilon_{d}$ is not increasing or if $\varepsilon_{d}$ is smaller than $x$; mathematically formulated as

$$
\begin{array}{llll}
\dot{D} \geqq 0 & \text { if } \quad \dot{\varepsilon}_{d}>0 \quad \text { and if } & \varepsilon_{d}=\varkappa, \\
\dot{D}=0 & \text { if } \quad \dot{\varepsilon}_{d} \leqq 0 \quad \text { or if } & \varepsilon_{d}<\varkappa .
\end{array}
$$


The actual value of the threshold $x$ can be expressed in the actual value of the damage $D$ as will be shown later on. For consistency it is required that

$\dot{\varkappa}=\left\langle\dot{\varepsilon}_{d}\right\rangle \quad$ if $\quad \varepsilon_{d}=\varkappa$,

$\dot{\varkappa}=0 \quad$ if $\quad \varepsilon_{d}<x$,

where the so-called McAuley brackets $\langle\cdot\rangle$ are defined by

$\langle\xi\rangle=\frac{1}{2}(\xi+|\xi|)$.

The set of strain tensors with $\varepsilon_{d}(\varepsilon)-\varkappa=0$, represented in strain space, constitutes the actual damage surface [13]; for strains mapped "inside" of this surface the material behaviour is reversible, only for strains "on" the surface damage growth may occur.

The damage evolution can be specified by the introduction of a mechanical dissipation potential [14]; more directly it is supposed here that damage growth is governed by a power-law

$\dot{D}=0 \quad$ if $\varepsilon_{d}<\varkappa$,

$\dot{D}=\alpha\left\langle\dot{\varepsilon}_{d}\right\rangle\left(\varepsilon_{d}\right)^{m}(1-D)^{-n} \quad$ if $\quad \varepsilon_{d}=\varkappa$

with $\alpha>0, m$ and $n>0$ material properties. The damage evolution equation expresses that the damage rate will become infinite if $D$ approaches the critical value 1 . The linearity with respect to $\dot{\varepsilon}_{d}$ corresponds with the previous restriction to time-independent material behaviour. Because $\dot{D} \geqq 0$ the entropy inequality is satisfied.

From the above formulation it can easily be observed that the damage $D$ and the threshold value $x$ are simultaneously increasing functions of the time $t$ (or are both constant); for irreversible as well as for reversible behaviour it can be written

$\dot{D}=\alpha \dot{x}(x)^{m}(1-D)^{-n}$.

With use of the initial values $D_{i}$ and $\varkappa_{i}$ for $D$ and $x$, respectively, the straightforward integration of this differential equation yields

$D(x)=1-\left(\left(1-D_{i}\right)^{n+1}-\frac{\alpha(n+1)}{m+1}\left((x)^{m+1}-\left(x_{i}\right)^{m+1}\right)\right)^{1 /(n+1)}$,

assuming $m \neq-1$; also for $m=-1$ an appropriate analytical expression can be derived, however, in the following this exceptional case will be left out of consideration.

By introducing of the critical threshold $\varkappa_{c}$, defined by $D\left(\varkappa_{c}\right)=1$, in the relationship (10) for $D(x)$, the quantity $\alpha$ can be written as

$\alpha=\frac{m+1}{n+1} \frac{\left(1-D_{i}\right)^{n+1}}{\left(x_{c}\right)^{m+1}-\left(x_{i}\right)^{m+1}}$.

Backsubstitution of this expression for $\alpha$ into Eq. (10) results in

$D(\varkappa)=1-\left(1-D_{i}\right)\left(\frac{\left(\varkappa_{c}\right)^{m+1}-(\varkappa)^{m+1}}{\left(\varkappa_{c}\right)^{m+1}-\left(\varkappa_{i}\right)^{m+1}}\right)^{1 /(n+1)}$.

The actual value of $x$ at time $t$ is completely determined by the history of the damage equivalent strain $\left\{\varepsilon_{d}(\tau) ; \tau \leqq t\right\}$ and consequently by the strain history $\{\boldsymbol{g}(\tau) ; \tau \leqq t\}$; it can be written

$\varkappa(t)=\operatorname{Max}\left[\varkappa_{i},\left\{\varepsilon_{d}(\tau) ; \tau \leqq t\right\}\right]$. 
If $\varepsilon_{d}$ exceeds $\varkappa_{c}$ the relationships derived above lose validity; the material is completely damaged then and $\boldsymbol{\sigma}$ should be set to 0 .

Resuming: for a given deformation history the damage $D$ and thus the stress tensor $\sigma$ can be calculated; besides the elastic properties $E$ and $v$ the damage quantities $D_{i}, x_{i}, x_{c}, m$ and $n$ should be specified; the relationship for $\varepsilon_{d}(\varepsilon)$ has to be supplied additionally. For the following elaborations the initial values of $D$ and $x$ are assumed to be negligible. The research will be continued with $D_{i}=0$ and $\varkappa_{i}=0$.

\section{Uniaxial stress}

For uniaxial loading the stress tensor $\boldsymbol{\sigma}$ can be written as $\sigma=\sigma_{a} e_{a} e_{a}$, with $\sigma_{a}$ the axial stress (positive for tension, negative for compression) and with $e_{a}$ the unit vector in axial direction. As a consequence of the invariance of Poisson's ratio the strain tensor $\varepsilon$ reads

$\varepsilon=\varepsilon_{a}\left(e_{a} e_{a}-v\left(\mathbf{I}-e_{a} e_{a}\right)\right)$

where $\varepsilon_{a}$ denotes the axial strain (positive for tension, negative for compression).

The equivalent damage strain $\varepsilon_{d}(\varepsilon)$ is scaled such that for uniaxial stress states $\varepsilon_{d}$ satisfies

$\varepsilon_{d}=\varepsilon_{a} \quad$ if $\quad \varepsilon_{a} \geqq 0$,

$\varepsilon_{d}=-\frac{\varepsilon_{a}}{\eta} \quad$ if $\quad \varepsilon_{a}<0$

where the material constant $\eta \geqq 1$ indicates the difference in damage sensitivity between tension and compression. For $\eta=1$ this difference is absent while for an increasing value of $\eta$ the compression strength becomes larger with respect to the tension strength.

If the axial strain is prescribed such that $\left|\varepsilon_{\alpha}\right|$ is monotonically increasing, the application of the theory developed leads to

$$
\begin{aligned}
& \sigma_{a}=E \varepsilon_{a}\left(1-\left(\frac{\varepsilon_{a}}{\varkappa_{c}}\right)^{m+1}\right)^{1 /(n+1)} \quad \text { if } \quad \varepsilon_{a} \geqq 0, \\
& \sigma_{a}=E \varepsilon_{a}\left(1-\left(\frac{-\varepsilon_{a}}{\eta \varkappa_{c}}\right)^{m+1}\right)^{1 /(n+1)} \quad \text { if } \quad \varepsilon_{a}<0 .
\end{aligned}
$$

From these equations the ratio of the extreme values of $\sigma_{a}$ in tension and compression can be derived

$\frac{\left|\sigma_{a}\right|_{\text {max in tension }}}{\left|\sigma_{a}\right|_{\max \text { in compression }}}=\frac{1}{\eta}$

while this ratio also holds for the associated values of $\left|\varepsilon_{a}\right|$. The intuitive assumption by Fonseka and Krajcinovic [15], $\eta=10$ for high strength concrete, will be adopted for the following elaborations. It is remarkable that with micromechanical calculations [16] almost the same value for $\eta$ has been found.

For a particular grade of concrete with $E=27.6 \cdot 10^{3}[\mathrm{MPa}]$ and a compression strength of 73.8 [MPa] experimental data for uniaxial compression [17] and theoretical results have been compared by Fonseka and Krajcinovic [15]. Using these publications the values of $\chi_{c}, m$ and $n$ for 
$\uparrow^{\left|\sigma_{\mathrm{a}}\right|[\mathrm{MPa}]}$

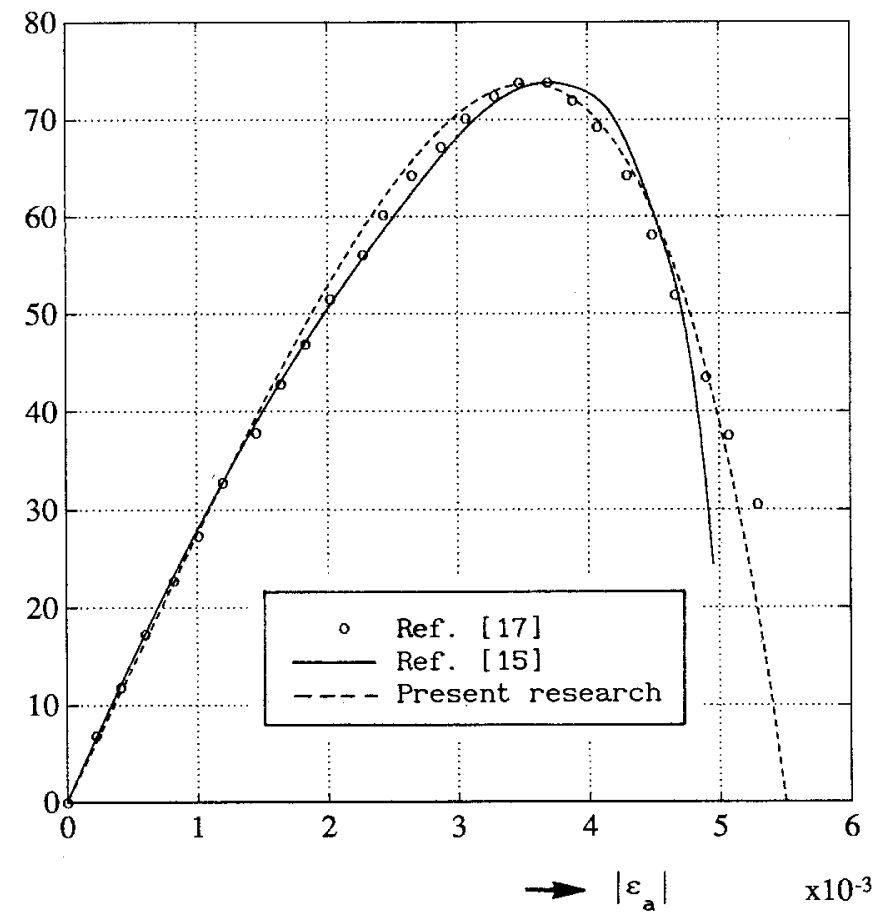

Fig. 1. Uniaxial compression

the present model are established: $x_{c}=0.00055, m=2$ and $n=0.1$. Figure 1 shows that the present approach is almost perfectly suitable to describe the experimental data and the results derived by Fonseka and Krajcinovic [15].

\section{Damage equivalent strain}

In the previous Section the scaling of the damage equivalent strain $\varepsilon_{d}$ has been defined by Eq. (15), taking into account the difference between tension and compression. An acceptable expression for $\varepsilon_{d}$, formulated in principal strains $\varepsilon_{j}(j=1,2,3)$, seems to be [18]

$\varepsilon_{d}=\gamma\left(\sum_{j=1}^{3}\left(\left\langle\varepsilon_{j}\right\rangle^{2}+h^{2}\left\langle-\varepsilon_{j}\right\rangle^{2}\right)\right)^{1 / 2}$

with $\gamma>0$ an adjustable multiplier and with $0 \leqq h \leqq 1$ a parameter to accomplish that compression is less harmful than tension because of closing cracks. For $h=1$ the distinction between tension and compression vanishes. The contribution $h^{2}\left\langle-\varepsilon_{j}\right\rangle^{2}$ is mostly omitted e.g. by Mazars [19] and Francois [20]. To meet the scaling conditions (15) $\gamma$ and $h$ should be related to $\eta$ by

$\gamma=\left(\frac{\eta^{2}-2 v^{2}}{\eta^{2}\left(1-4 v^{4}\right)}\right)^{1 / 2}, \quad h=\left(\frac{1-2 v^{2} \eta^{2}}{\eta^{2}-2 v^{2}}\right)^{1 / 2}$

It is evident that for $\eta>1 /(v \sqrt{2})$, which may be quite realistic (see Section 3), the definition (18) for $\varepsilon_{d}$ fails. Therefore an alternative relationship for $\varepsilon_{d}$ is proposed here. 
Replacement of principal strains in Eq. (18) by principal stresses leads to a more suitable expression for $\varepsilon_{d}$. The result can be written as

$\varepsilon_{d}=\bar{\gamma}\left(\sum_{j=1}^{3}\left(\left\langle\lambda_{j}\right\rangle^{2}+\bar{h}^{2}\left\langle-\lambda_{j}\right\rangle^{2}\right)\right)^{1 / 2}$

with $\lambda_{j}(j=1,2,3)$ linear combinations of $\varepsilon_{j}(j=1,2,3)$ according to

$\left[\begin{array}{l}\lambda_{1} \\ \hat{\lambda}_{2} \\ \lambda_{3}\end{array}\right]=\left[\begin{array}{lll}1-v & v & v \\ v & 1-v & v \\ v & v & 1-v\end{array}\right]\left[\begin{array}{l}\varepsilon_{1} \\ \varepsilon_{2} \\ \varepsilon_{3}\end{array}\right]$.

By scaling with (15), the quantities $\bar{\gamma}$ and $\bar{h}$ are expressed in $v$ and $\eta$

$\bar{\gamma}=\frac{1}{(1+v)(1-2 v)}, \quad \bar{h}=\frac{1}{\eta}$.

Using the damage equivalent strain defined by Eqs. (20)-(22), there are no difficulties to deal with large values of the parameter $\eta$. Therefore this strain measure will be applied in Section 6 to analyse a concrete softening problem.

\section{Finite element method}

A continuum is considered, subjected to (increasing) external loads and/or prescribed displacements. At any stage of the loading process it is required that

- the stress field satisfies the local equilibrium equation,

- the local stress tensor $\sigma$ and the damage variable $D$ are related to the local strain tensor $\varepsilon$ and the strain history through the constitutive relationships as developed in Section 2,

- the kinematic and dynamic boundary conditions are satisfied in conformity with the process specification.

Because of history dependence an incremental approach will be followed: the calculation of the interesting quantities is executed for a discrete number of subsequent process stages. A suitable numerical procedure will be necessary for the analysis on increment level, to deal with the inhomogeneous character of the relevant fields. The finite element method is generally accepted as the appropriate solution technique.

To manipulate the differential problem formulation, the displacement field $\boldsymbol{u}(\boldsymbol{x})$, with $\boldsymbol{x}$ the position vector of the material points, is approximated by the kinematically admissible discretization

$\boldsymbol{u}(\boldsymbol{x})=\underset{\sim}{N^{T}}(\boldsymbol{x}) \underset{\sim}{\boldsymbol{u}}$.

The nodal displacement vectors constitute the column $\underset{\sim}{\boldsymbol{u}}$, the column $\underset{\sim}{N}(x)$ contains the interpolation functions; superscript $T$ denotes transposition. A Galerkin approach, applied to the weak formulation of the equilibrium equation [21], results in the balance of nodal forces

$\underset{\sim}{f_{i}}(\underset{\sim}{\boldsymbol{u}})=\boldsymbol{f}_{e}$.

The column $f_{i}$ with internal nodal forces is defined by

$f_{i}(\underline{u})=\int_{V}[\nabla N] \cdot \sigma d V$ 
with $V$ the volume of the continuum and $\boldsymbol{V}$ the gradient operator in that volume. With the constitutive relationships the stress tensor $\boldsymbol{\sigma}$ is non-linearly (due to the damage contribution) expressed in the strain tensor $\boldsymbol{\varepsilon}$ and thereupon in the column $\underset{\sim}{\boldsymbol{u}}$ with nodal displacements, which are related by

$\varepsilon=\frac{1}{2}\left[[\nabla \underset{\sim}{N}]+[\nabla \underset{\sim}{N}]^{T}\right] \underset{\sim}{\boldsymbol{u}}$

The column ${\underset{\sim}{e}}_{e}$ with external nodal forces is defined by

$f_{e}=\int_{A} \underset{\sim}{N} p d A$

with $\boldsymbol{p}$ the stress vector acting on the boundary $A$; in $\boldsymbol{f}_{e}$ the dynamic boundary conditions are taken into account.

Formally, the non-linearity in the system equations (24) necessitates an iterative solution scheme on increment level. If the standard Newton-Raphson method [21] is applied (but also for alternative algorithms), the implementation of a consistent tangential stiffness matrix $d \boldsymbol{f}_{i} / d \underline{u}$ is advantageous for numerical convergence. However, as a consequence of the incremental approach, combined with the softening material behaviour, bifurcation phenomena may occur, causing convergence and ambiguity problems (P. Fotiu [22] studied the convergence problem and found unconditionally stable algorithms). To avoid these, the behaviour during an increment is assumed linear by fixation of the damage field. Then the solution of the nodal displacements can directly (without iterations) be determined. At the end of each increment first the damage field and next the stress field are updated using Eqs. (12) and (1). The final incremental result will show an unbalance (residual load) with respect to Eq. (24). If the error exceeds a certain small limit value one or more so-called "zero-increments" (incremental steps with unchanged boundary conditions) are executed until equilibrium is restored. Especially for ill-conditioned systems, the "uncoupled" procedure outlined above proves to be advantageous.

\section{Example}

The uniaxial compression of a rectangular concrete block under plane strain conditions is considered, see Fig. 2.

The boundaries $x= \pm b e_{1}$ are stress-free, for the boundaries $x= \pm h e_{2}$ the displacement in $e_{1}$-direction is suppressed while in $\boldsymbol{e}_{2}$-direction the displacement is prescribed according to $\boldsymbol{u}\left(\boldsymbol{x}= \pm h \boldsymbol{e}_{2}\right)= \pm \delta h e_{2}$ (constrained compression). With $\delta<0$ the dimensionless height reduction (average strain in $e_{2}$-direction) is indicated; $|\delta|$ increases monotonically.

The material properties are taken from Section 3, additionally $v=0.2$. The numerical calculation is performed for the geometry ratio $b / h=0.5$. A quarter of the configuration is analysed, using a regular mesh of $8 \times 8$ isoparametric four-node elements with a bilinear displacement field and four integration points.

Figure 3 shows the average upsetting pressure $p$ (positive in case of compression) as a function of $|\delta|$. As a reference also the graph for unconstrained uniaxial plane strain compression is visualized, obeying the relationship

$p=\frac{E|\delta|}{1-v^{2}}\left(1-\left(\frac{\varepsilon_{d}}{\chi_{c}}\right)^{m+1}\right)^{1 /(n+1)}, \quad \varepsilon_{d}=\frac{1}{\eta} \frac{\left(1+v^{2}\right)^{1 / 2}}{1-v^{2}}|\delta|$ 


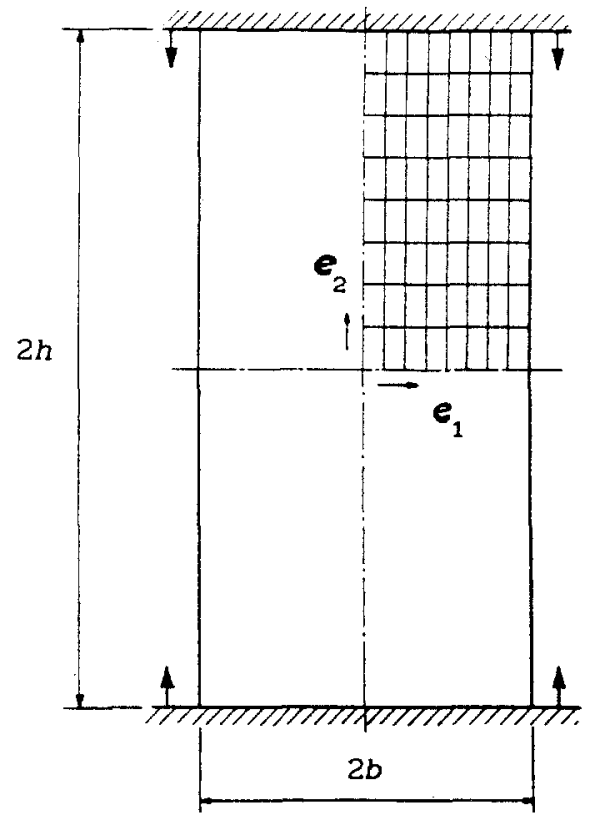

Fig. 2. Problem configuration and finite element mesh

$p[\mathrm{MPa}]$

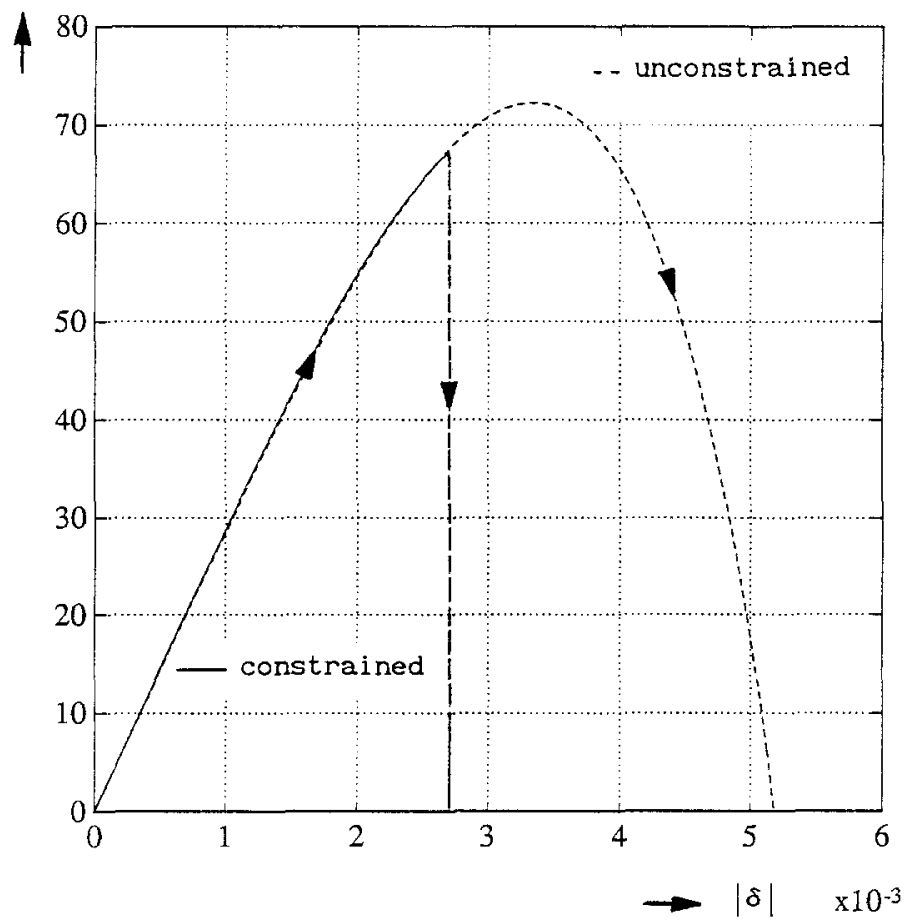

Fig. 3. Upsetting pressure 


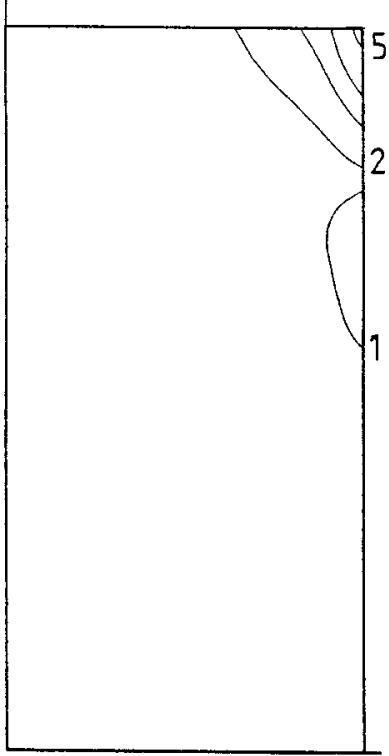

level 1: $D=0.1$

step : $\Delta D=0.1$

level $5: D=0.5$

Peak pressure

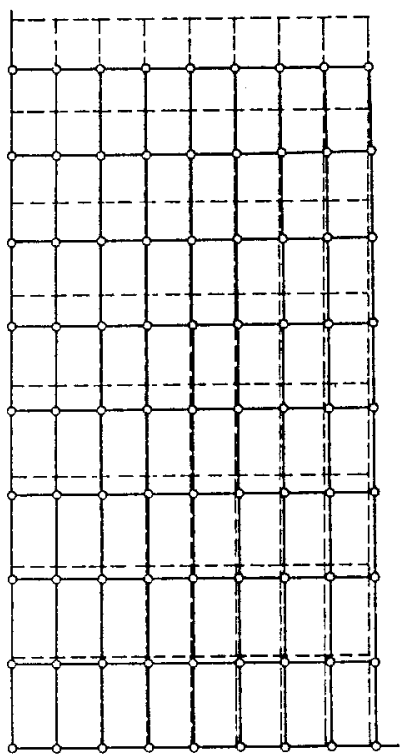

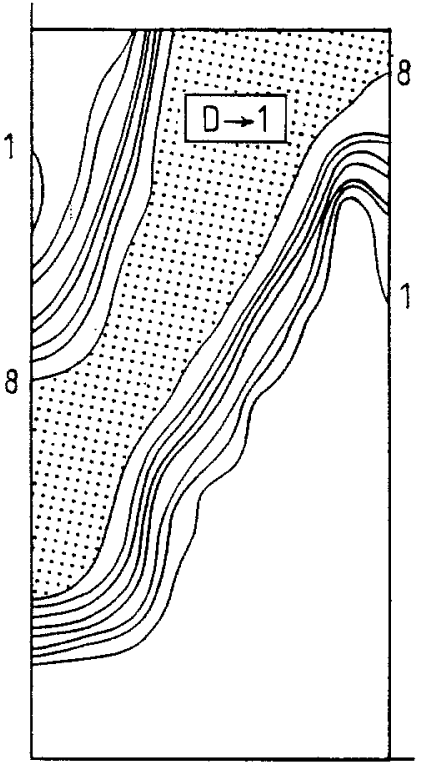

level $1: D=0.2$

step: $\Delta D=0.1$

level $8: D=0.9$

Final state

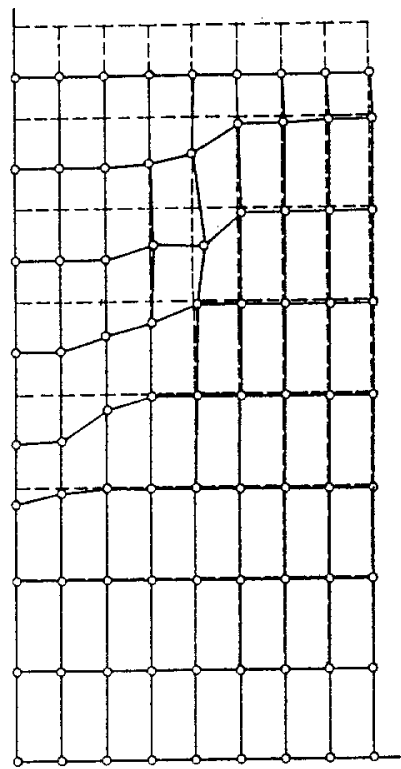

Fig. 4. Damage and deformation for constrained uniaxial compression

to be derived easily from the theoretical framework. For unconstrained compression the post-peak pressure decreases gradually, due to the implicit assumption that the configuration remains homogeneous. Without this assumption the solution is non-uniquely determined [23]. For constrained compression the post-peak pressure almost immediately drops down, following 
a non-equilibrium path; for low pressure equilibrium is restored. The differences can be explained by

- the heterogeneity in the constrained case combined with the softening material behaviour leading to unstable localization of damage,

- the presence, in the constrained case, of tensile principal stresses which have a dominating effect on the damage equivalent strain $\varepsilon_{d}$.

Figure 4 shows for the constrained case the contours of constant damage and the deformed element mesh (displacements multiplied by 25 ) at peak pressure and in the final state. At peak pressure the damage is mainly concentrated in the corners $x= \pm b e_{1} \pm h e_{2}$, as can be expected. Then, from the corners the damage propagates to the centre of the material; shear band formation can clearly be observed. The damage development is according to the pattern well-known from practical experience.

\section{Concluding remarks}

Continuum damage mechanics proves to be a powerful tool to analyse the behaviour of brittle materials in the softening regime. The constitutive equation for the undamaged case is extended with a damage contribution. Essentially therefore, the damage criterion and the damage evolution law have to be specified. To deal with large differences in tension and compression strength an appropriate formulation for the damage equivalent strain in the damage criterion has been proposed. The damage evolution law has been quantified with a simple expression supported by experimental results.

The numerical elaboration of the theoretical framework for inhomogeneous configurations can be performed with the finite element method. The history dependence requires an incremental approach. To avoid convergence and bifurcation problems an uncoupled strategy has been chosen: per increment only a linear system of equations has to be solved, however, the increment size is considerably limited to obtain a prescribed accuracy. So-called zero-increments are executed to maintain equilibrium of internal and external nodal forces. It is emphasized that the solution technique should be given extra attention in future research. Nevertheless, the present method was capable to produce interesting results for constrained uniaxial compression of concrete under plane strain conditions.

\section{References}

[1] Kachanov, L. M.: Time of the rupture process under creep conditions. Tzv. Akad. Nauk. SSR. Otd. Tekh. 8, 26-31 (1958).

[2] Becker, W., Gross, D.: A one-dimensional micromechanical model of elastic-microplastic damage evolution. Acta Mech. 70, $221-233$ (1987).

[3] Malvern, L. E.: Introduction to the mechanics of a continuous medium. Englewood Cliffs: Prentice-Hall 1969.

[4] Müller, I.: Thermodynamics. London: Pitman 1984.

[5] Germain, P., Nguyen, Q. S., Suquet, P.: Continuum thermodynamics. J. Appl. Mech. 50, 1010-1020 (1983).

[6] Kestin, J., Bataille, J.: Irreversible thermodynamics of continua and internal variables. Continuum models of discrete systems. J. W. Provan, University of Waterloo Press 1978.

[7] Chaboche, J. L.: Continuum damage mechanics. Parts 1 and 2. J. Appl. Mech. 55, $59-72$ (1988). 
[8] Billardon, R., Moret-Bailly, L.: Fully coupled strain and damage finite element analysis of ductile fracture. Nucl. Engng. Design 105, $43-49$ (1987).

[9] Lemaitre, J.: Local approach of fracture. Engng. Fract. Mech. 25, 523-537 (1986).

[10] Kachanov, L. M.: Introduction to continuum damage mechanics. Dordrecht: Martinus Nijhoff 1986.

[11] Simo, J. C., Ju, J. W.: Strain- and stress-based continuum damage models. Parts 1 and 2. Int. J. Solids Struct. 23, $821-869$ (1987).

[12] Lai, W. H., Rubin, D., Krempl, E.: Introduction to continuum mechanics. Pergamon Press 1979.

[13] Krajcinovic, D., Fonseka, G. U.: The continuous damage theory of brittle materials. Part 1. General theory. J. Appl. Mech. 48, 809-815 (1981).

[14] Lemaitre, J., Chaboche, J. L.: Mécanique des matériaux solides. Paris: Dunod, Bordas 1988.

[15] Fonseka, G. U., Krajcinovic, D.: The continuous damage theory of brittle materials. Part 2. Uniaxial and plane response modes. J. Appl. Mech. 48, 816-824 (1981).

[16] Vonk, R. A., Rutten, H. S., van Mier, J. G. M., Fijneman, H. J.: Micromechanical simulation of concrete softening. Proc. Fracture Processes in Brittle Disordered Materials, Noordwijk, The Netherlands, preprint (1991).

[17] Wang, P. T.: Complete stress-strain curve of concrete and its effect on ductility of reinforced concrete members. Ph. D. Thesis, University of Illinois at Chicago Circle 1977.

[18] Paas, M. H. J. W.: Continuum damage mechanics with an application to fatigue. Ph. D. Thesis, Eindhoven University of Technology, The Netherlands 1990.

[19] Mazars, J.: Probabilistic aspects of mechanical damage in concrete structures. Proc. Int. Conf. on Fracture Mechanics Technology applied to Material Evaluation and Structural Design, Melbourne, Australia, pp. $657-666$ (1982).

[20] Francois, D.: Fracture and damage. In: Elastic-plastic fracture mechanics (Larsson, L. H., ed.), pp. 285-302. Dordrecht: D. Reidel Publishing Company 1985.

[21] Bathe, K. J.: Finite element procedures in engineering analysis. Englewood Cliffs: Prentice-Hall 1982.

[22] Fotiu, P.: Die dynamische Wirkung dissipativer Prozesse in Materialien mit Mikrodefekten mit Anwendungen auf Schwingungsprobleme inelastischer Plattentragwerke. Dissertation, TU Wien 1990.

[23] Bazant, Z. P., Pijaudier-Cabot, G.: Nonlocal continuum damage, localization instability and convergence. J. Appl. Mech. 55, 287-293 (1988).

Authors' address: W. A. M. Brekelmans, P. J. G. Schreurs and J. H. P. de Vree, Mechanical Engineering Department, University of Technology, P.O. Box 513, Eindhoven, The Netherlands 